\title{
The Ginzburg-Landau equation III. Vortex dynamics
}

\author{
Yu N Ovchinnikov† and I M Sigal $§$ \\ $\dagger$ Landau Institute, Moscow, Russia \\ $\ddagger$ Department of Mathematics, University of Toronto, Toronto, Canada M5S 3G3
}

Received 6 May 1997, in final form 6 March 1998

Recomended by P Constantin

\begin{abstract}
In this paper we study the time-dependent Ginzburg-Landau equation of the Schrödinger type in two dimensions. The initial conditions are chosen to describe several well-separated vortices. Our task is to understand the vortex structure of the corresponding solutions as well as corrections due to radiation. To this end we develop the nonlinear adiabatic theory. Using the methods of effective action and of geometric solvability we derive equations for the vortex dynamics and radiation. As an example we consider the special case of radiation by two 1 -vortices.
\end{abstract}

AMS classification scheme numbers: 35Q55, 70K99

\section{Introduction}

The nonlinear Schrödinger equation is one of the simplest equations which has particlelike solutions-solitons, kinks and vortices-as well as wave-like excitations. A general solution, at least for large times, can be described in terms of a collection of moving localized structures-each of which looks like a particle-like solution centred at some point-interacting between themselves as well as with wave excitations. This leads to a rather complex dynamics. It is the goal of this paper to study this dynamics for the nonlinear Schrödinger equation

$$
\mathrm{i} \frac{\partial \psi}{\partial t}=-\Delta \psi+\left(|\psi|^{2}-1\right) \psi
$$

on $\mathbb{R}_{x}^{2} \times \mathbb{R}_{t}^{1}$, where $\Delta$ is the Laplacian in the spatial variable $x \in \mathbb{R}^{2}$ and for each $t, \psi(\cdot, t)$ : $\mathbb{R}^{2} \rightarrow \mathbb{C}$, and satisfies the boundary condition

$$
|\psi(x, t)| \rightarrow 1 \quad \text { as }|x| \rightarrow \infty .
$$

This equation is called the Gross-Pitaevski equation. It comes up in condensed matter physics and nonlinear optics. The steady state equation corresponding to equation (1.1) is the celebrated Ginzburg-Landau equation,

$$
-\Delta \psi+\left(|\psi|^{2}-1\right) \psi=0 .
$$

It is subject to the boundary condition

$$
|\psi| \rightarrow 1 \quad \text { as }|x| \rightarrow \infty .
$$

$\S$ E-mail: sigal@math.toronto.edu 
Let $(r, \theta)$ be the polar coordinates of $x$. For each integer $n$, the latter equation has a solution of the form (modulo translations and rotations)

$$
\psi^{(n)}(x)=f^{(n)}(r) \mathrm{e}^{\mathrm{i} n \theta},
$$

where $f^{(n)}(r)$ is a unique function monotonically increasing from 0 to 1 as $r$ runs from 0 to $\infty$ (see, e.g. [BBH, H, OSa]). Such solutions are called $n$-vortices.

In this paper we study equation (1.1) with initial conditions which describe roughly several, say $K$, well-separated vortices. A precise definition will be given later; for now we just present an example of such an initial condition:

$$
\psi_{\text {indep }}(x)=\prod_{i=1}^{K} \psi^{\left(n_{i}\right)}\left(x-a_{i}\right)
$$

with $a \equiv \min _{i \neq j}\left|a_{i}-a_{j}\right| \gg 1$. The goal of this paper is to understand the dynamics of the vortices dominating the solution $\psi$ to equations (1.1) and (1.2). We show that in the leading order in $a^{-1}$ the dynamics of vortex centres is described by the Hamiltonian system

$$
\dot{\boldsymbol{a}}=J \nabla E(\boldsymbol{a}),
$$

where $\boldsymbol{a}=\left(a_{1}, \ldots, a_{K}\right), a_{j} \in \mathbb{R}^{2}, J$ is the block-diagonal matrix with the blocks $\frac{1}{\pi n_{i}}\left(\begin{array}{cc}0 & 1 \\ -1 & 0\end{array}\right), i=1, \ldots, K$, and $E(\boldsymbol{a})$ is the intervortex energy introduced in [OSb] (see section 2). It is shown in [OSb] that

$$
E(\boldsymbol{a})=\Sigma E_{n_{i}}+H(\boldsymbol{a})+\mathrm{O}\left(\frac{1}{a}\right),
$$

where $E_{n}$ is the proper energy of the $n$-vortex (see section 2 for precise definitions and a sketch of the proof of (1.8)) and $H(\boldsymbol{a})$ is the Kirchoff-Onsager Hamiltonian given by

$$
H(\boldsymbol{a})=-\pi \sum_{i \neq j} n_{i} n_{j} \ln \left|a_{i j}\right|
$$

with $a_{i j}=a_{i}-a_{j}$. Thus, in the leading order we recover the Kirchoff-Onsager law

$$
\dot{a}=J \nabla H(a),
$$

which was pointed out in $[\mathrm{O}, \mathrm{CM}, \mathrm{G}]$ and derived using multiple scale expansion in $[\mathrm{N}]$.

We argue that in the next order the contribution is due to the interaction of the vortices with radiation produced by the vortex motion. We give a general estimate of this contribution and then present an explicit result in the special case of two 1-vortices. The radiation phenomenon in this context seems not to have been observed previously.

To derive (1.7) we develop an approach which we call the nonlinear adiabatic theory. It goes roughly as follows (cf [AH, Ma, Stu]). Let $S(\psi)$ be the action functional for equation (1.1):

$$
S(\psi)=\int\left\{-\int \frac{1}{2} \operatorname{Im}(\psi \dot{\bar{\psi}}) \mathrm{d}^{2} x+\mathcal{E}_{\text {ren }}(\psi)\right\} \mathrm{d} t,
$$

where $\mathcal{E}_{\text {ren }}(\psi)$ is the renormalized Ginzburg-Landau functional (see section 2). First we find an approximate minimizer, $\psi_{a}$, of $\mathcal{E}_{\text {ren }}(\psi)$ under the constraint that the vortices are fixed at positions $a_{1}, \ldots, a_{k},\left(a_{1}, \ldots, a_{k}\right)=\boldsymbol{a}$. Next, we allow $\boldsymbol{a}$ to depend on time and plug $\psi_{\boldsymbol{a}(t)}$ into (1.11). The resulting action functional,

$$
S_{\mathrm{eff}}(\boldsymbol{a}) \equiv S\left(\psi_{a}\right),
$$


describes the dynamics of the vortex centres in the leading approximation; it is equal modulo $\int \mathrm{O}\left(\ln a \cdot a^{-2}\right) \mathrm{d} t$ to the action functional

$$
S_{\mathrm{vort}}(\boldsymbol{a})=\int\left\{-\frac{\pi}{2} \sum_{j=1}^{k} n_{j} a_{j} \wedge \dot{a}_{j}-E(a)\right\} \mathrm{d} t,
$$

whose critical points satisfy equation (1.7).

To go beyond this theory we write $\psi=\psi_{a}+\alpha$, where $\alpha$ is supposed to be a small fluctuation field around $\psi_{a}$ and expand $S(\psi)$ in $\alpha$ up to the second order. Critical points of the resulting functional satisfy the system of coupled equations

$$
\begin{aligned}
& \partial_{\boldsymbol{a}} S_{\mathrm{eff}}(\boldsymbol{a})=-\nabla_{\boldsymbol{a}} \operatorname{Re} \int \bar{\alpha} \partial_{\bar{\psi}} S\left(\psi_{\boldsymbol{a}}\right) \\
& S^{\prime \prime}\left(\psi_{\boldsymbol{a}}\right) \alpha=-\partial_{\bar{\psi}} S\left(\psi_{\boldsymbol{a}}\right),
\end{aligned}
$$

where $\partial_{\varphi}$ stands for the variational derivative with respect to $\varphi$ and $S^{\prime \prime}(\psi)$ is the Hessian of $S$ at $\psi$, both defined in sections 2 and 4, respectively, and where we dropped the higher-order term $\nabla_{a} \frac{1}{2} \operatorname{Re} \iint \bar{\alpha} S^{\prime \prime}\left(\psi_{a}\right) \alpha$. In section 6 we demonstrate that provided $\boldsymbol{a}$ satisfies (1.7), one can perturb $\psi_{a}$ slightly in such a way that equation (1.15) has a solution of the order $\alpha=\mathrm{O}\left(a^{-1}\right)$, provided $t \leqslant a^{p}$ for some $p \geqslant 0$. To this end we decompose the space $\mathbb{R}^{2}$ into several regions determined by the configurations $\boldsymbol{a}$ and estimate equation (1.15) separately in each region. We call this method, the method of geometric solvability.

The analysis of the previous paragraph can be summed up by showing that the solution $\psi$ of (1.1) and (1.2) for $t \leqslant a^{p}$ for some $p \geqslant 0$ for an appropriate initial condition is of the form

$$
\psi=\psi_{a(t)}+\alpha
$$

where $\psi_{\boldsymbol{a}}$, an approximate minimizer of $\mathcal{E}_{\text {ren }}$, has zeros at $a_{1}, \ldots, a_{K}, \boldsymbol{a}(t)$ satisfies (1.7), and $\alpha$ (as well as $\nabla_{x} \alpha$ ) is of order $\mathrm{O}\left(a^{-1}\right)$. Then implicit function theorem implies that $\psi$ has zeros at the positions

$$
a(t)+\mathrm{O}\left(a^{-1}\right)
$$

with the corresponding vorticities. Thus, equation (1.7) describes the vortex dynamics for time up to $a^{p}$ for some $p \geqslant 0$ to a rather high precision.

We observe that equations (1.14)-(1.15) stripped of inessential terms read

$$
\dot{\boldsymbol{a}}=J \nabla H(\boldsymbol{a})+2 \nabla_{\boldsymbol{a}} \int \varphi_{0} \ddot{\chi}
$$

and

$$
\left(\partial_{t}^{2}-2 \Delta_{x}\right) \chi=-4 \ddot{\varphi}_{0}
$$

where $\chi=$ phase $\alpha-\varphi_{0}$ and $\varphi_{0}(x)=\sum_{j=1}^{K} n_{j} \theta\left(x-a_{j}\right), \theta(x)=$ polar angle of $x$. These equations describe motion of interacting vortex centres coupled to radiation.

Finally, the dynamics of vortices for the heat equation was rigorously derived in [Lin] and for the abelian Higgs model in the near critical regime, in [Stu] (see also [AH, Ma, $\mathrm{Sh}])$. In what follows the time derivative $\frac{\partial \psi}{\partial t}$ is often denoted as $\dot{\psi}$. 


\section{Renormalized Ginzburg-Landau energy}

Solutions of equations (1.1) and (1.2) (or (1.3) and (1.4)) are classified by the total index (winding number) of $\psi$, considered as a vector field on $\mathbb{R}^{2}$, at $\infty$, i.e.

$$
\operatorname{deg} \psi:=\frac{1}{2 \pi} \int_{|x|=R} \mathrm{~d}(\arg \psi)
$$

for $R$ sufficiently large. We call this index (as opposed to local indices of $\psi$ considered below, see equation (2.8)) the degree (or total vorticity) of $\psi$.

It is a straightforward observation that equation (1.3) is the equation for critical points of the following functional

$$
\mathcal{E}(\psi)=\frac{1}{2} \int\left(|\nabla \psi|^{2}+\frac{1}{2}\left(|\psi|^{2}-1\right)^{2}\right)
$$

Indeed, if we define the variational derivative, $\partial_{\psi} \mathcal{E}(\psi)$, of $\mathcal{E}$ by

$$
\operatorname{Re} \int \xi \partial_{\psi} \mathcal{E}(\psi)=\left.\frac{\partial}{\partial \lambda} \mathcal{E}\left(\psi_{\lambda}\right)\right|_{\lambda=0}
$$

for any path $\psi_{\lambda}$ s.t. $\psi_{0}=\psi$ and $\left.\frac{\partial}{\partial \lambda} \psi_{\lambda}\right|_{\lambda=0}=\xi$, then the 1.h.s. of equation (1.1) is equal to $\overline{\partial_{\psi} \mathcal{E}(\psi)}=\partial_{\bar{\psi}} \mathcal{E}(\psi)$ for $\mathcal{E}(\psi)$ given in (2.1).

Equation (2.1) is the celebrated Ginzburg-Landau (free) energy. However, there is a problem with it in our context. It is shown in [OSa] that if $\psi$ is an arbitrary $C^{1}$ vector field on $\mathbb{R}^{2}$ s.t. $|\psi| \rightarrow 1$ as $|x| \rightarrow \infty$ and $\operatorname{deg} \psi \neq 0$, then $\mathcal{E}(\psi)=\infty$.

We renormalize the Ginzburg-Landau energy functional as follows (see [OSa]). Let $\chi(x)$ be a smooth real function on $\mathbb{R}^{2}$ s.t.

$$
\chi(x)= \begin{cases}1 & \text { for }|x| \geqslant 2 \\ 0 & \text { for }|x| \leqslant 1\end{cases}
$$

Define

$$
\mathcal{E}_{\text {ren }}(\psi)=\frac{1}{2} \int\left(|\nabla \psi|^{2}-\frac{(\operatorname{deg} \psi)^{2}}{r^{2}} \chi+F\left(|\psi|^{2}\right)\right) \mathrm{d}^{2} x
$$

where

$$
F(u)=\frac{1}{2}(u-1)^{2} .
$$

We list here the most important properties of $\mathcal{E}_{\text {ren }}(\psi)$ (see [OSa] for the proofs):

(a) $\partial_{\bar{\psi}} \mathcal{E}_{\text {ren }}(\psi)=-\Delta \psi+F^{\prime}\left(|\psi|^{2}\right) \psi$;

(b) given $n$ let $M_{n}=\left\{\psi=f \mathrm{e}^{\mathrm{i} \varphi}\left|\int_{|x| \geqslant 2} \frac{1}{r^{2}}\right| 1-f^{2} \mid<\infty, f\right.$ is continuous and $f(0)=0$, $\int|\nabla(\varphi-n \theta)| r^{-1}<\infty$ and $\left.\int|\nabla(\varphi-n \theta)|^{2}<\infty\right\}$. Then $\mathcal{E}_{\text {ren }}(\psi)<\infty \forall \psi \in M_{n}$.

(c) We have the following bound from below

$$
\mathcal{E}_{\text {ren }}(\psi) \geqslant \mathcal{E}_{B(0,2)}(\psi)+\frac{1}{2} \int_{|x| \geqslant 2}\left(|\nabla| \psi||^{2}-\frac{1}{2}|\nabla \varphi|^{4}\right) \mathrm{d}^{2} x,
$$

where $\varphi=\arg \psi$, and for $\Omega \subset \mathbb{R}^{2}$,

$$
\mathcal{E}_{\Omega}(\psi)=\frac{1}{2} \int_{\Omega}\left(|\nabla \psi|^{2}-\frac{(\operatorname{deg} \psi)^{2}}{r^{2}} \chi+F\left(|\psi|^{2}\right)\right) \mathrm{d}^{2} x .
$$

It is known that $\psi^{(n)}$ are local minima for $n=0, \pm 1$ and are saddle points for $|n| \geqslant 2$ (see [OSa, M1,LL]). Moreover, $\psi^{(n)}$ with $n=0, \pm 1$ are the only local minima of $\mathcal{E}_{\text {ren }}(\psi)$ 
[M2, CK], while as far as saddle points are concerned one expects that there are other solutions breaking the rotational symmetry [OSc].

Consider once-differentiable functions $\psi: \mathbb{R}^{2} \rightarrow \mathbb{C}$ satisfying $|\psi| \rightarrow 1$ as $|x| \rightarrow \infty$. Let $\boldsymbol{a}=\left(a_{1}, \ldots, a_{m}\right)$ and $\boldsymbol{n}=\left(n_{1}, \ldots, n_{m}\right)$, where $a_{j} \in \mathbb{R}^{2}$ and $n_{j} \in \mathbb{Z}, j=1, \ldots, m$. $\boldsymbol{c}=(\boldsymbol{a}, \boldsymbol{n})$ will be called the vortex configuration. We say that $\psi$ has the vortex configuration $\boldsymbol{c}$ and write $\operatorname{conf} \psi=\boldsymbol{c}$, if $\psi$ has zeros (only) at $a_{1}, \ldots, a_{m}$ with local indices $n_{1}, \ldots, n_{m}$, respectively, i.e.

$$
\int_{\gamma_{j}} \mathrm{~d}(\arg \psi)=2 \pi n_{j}
$$

for any contour $\gamma_{j}$ containing $a_{j}$, but not the other zeros of $\psi$ and for $j=1, \ldots, m$. Now we define

$$
E(\boldsymbol{c})=\inf \left\{\mathcal{E}_{\text {ren }}(\psi) \mid \operatorname{conf} \psi=c\right\}
$$

(cf $[\mathrm{BBH}]$, for topological variational problems see [FS]). By property (c) above, $E(c)>$ $-\infty$. We call $E(c)$ the energy of the vortex configuration $c$. It will play a central role in our analysis. Usually, $\boldsymbol{n}$ is fixed during our considerations and we write $E(\boldsymbol{a})$ for $E(\boldsymbol{c})$, $\boldsymbol{c}=(\boldsymbol{a}, \boldsymbol{n})$.

In $[\mathrm{OSb}]$ we demonstrate the following asymptotic expression

$$
E(\boldsymbol{a})=\sum_{i=1}^{K} E_{n_{i}}+H(\boldsymbol{a})+\mathrm{O}\left(\frac{1}{a}\right)
$$

where $E_{n}=\mathcal{E}_{\text {ren }}\left(\psi^{(n)}\right)$, the self-energy of the $n$-vortex, and $H(\boldsymbol{a})$ is the function given in (1.8).

We sketch here the derivation of (2.10). For a complete demonstration see [OSb]. The upper bound, $E(c) \leqslant$ r.h.s. (2.10), is obtained by choosing an appropriate test function, $\psi$, $\operatorname{conf} \psi=c$, and performing a rather delicate many-body geometric analysis in order to show that $\mathcal{E}_{\text {ren }}(\psi)=$ r.h.s. (2.10). Then the bound itself follows from the variational inequality $E(c) \leqslant \mathcal{E}_{\text {ren }}(\psi)$. The function $\psi$ is described by the relations

$$
\psi=\psi_{j} \mathrm{e}^{\mathrm{i} \varphi_{(j)}}+\mathrm{O}\left(\frac{1}{\left|x-a_{j}\right| \cdot a}\right),
$$

for $\left|x-a_{j}\right| \ll a$ and $\forall j$, and

$$
\psi=\mathrm{e}^{\mathrm{i} \varphi_{0}}+\mathrm{O}\left(d(x, \boldsymbol{a})^{-2}\right)
$$

for $d(x, \boldsymbol{a}) \gg 1$. Here $d(x, \boldsymbol{a})=\min _{k}\left|x-a_{k}\right|, \psi_{j}(x):=\psi^{\left(n_{j}\right)}\left(x-a_{j}\right)$, and

$$
\varphi_{(j)}=\sum_{k, k \neq j} \varphi_{k} \quad \text { and } \quad \varphi_{0}=\sum_{k} \varphi_{k}
$$

with $\varphi_{j}(x):=\arg \psi_{j}(x)=n_{j} \theta\left(x-a_{j}\right)$, where $\theta(x)$ is the polar angle of $x$.

An example of a function $\psi$ satisfying (2.11) and (2.12) is the field describing independent vortices

$$
\psi_{\text {ind }}(x)=\prod_{j=1}^{K} \psi_{j}(x) .
$$

To see this one uses the estimate $\psi^{(n)}(x)=\mathrm{e}^{\mathrm{i} n \theta(x)}\left(1+\mathrm{O}\left(|x|^{-2}\right)\right)$. Another example is given by a field describing pinned vortices (e.g. solving equation (2.20) below), described below.

The geometric analysis mentioned above consists of breaking up the integral in (2.4) into a sum of the integrals over the disks $D_{j}=\left\{x \in \mathbb{R}^{2}|| x-a_{j} \mid \leqslant r_{0}\right\}, j=1, \ldots, K$, 
with $r_{0}=\mathrm{O}(a)$ but $\ll a$ and the integral over the rest of the space $D_{0} \equiv \mathbb{R}^{2} \backslash \bigcup_{j} D_{j}$, and estimating it separately in each $D_{j}, j=0, \ldots, K$, using (2.11) and (2.12).

In order to prove the lower bound, $E(c) \geqslant$ r.h.s. (2.10), we introduce the energy functional incorporating defects whose purpose is to pin the vortices down:

$$
\mathcal{E}_{\lambda}(\psi)=\mathcal{E}_{R}(\psi)+\Sigma \frac{1}{2} \lambda_{j} \int \delta_{b_{j}}|\psi|^{2},
$$

where $\lambda=\left(\lambda_{1}, \ldots, \lambda_{K}\right), \lambda_{j}>0$, are coupling constants of the defects and $\delta_{b_{j}} \geqslant 0$ are their potentials, centred at points $b_{j} \in \mathbb{R}^{2}$. We take $\delta_{b}$ to be either

$$
\delta_{b}=\frac{1}{2 \pi \bar{r}} \delta(|x-b|-\bar{r}),
$$

where $\bar{r}=\mathrm{O}(1)$, or a smooth version of this, i.e. $\delta_{b}$ is a smooth function supported in the annulus

$$
\left\{x \in \mathbb{R}^{2}|\bar{r} \leqslant| x-b \mid \leqslant \bar{r}+\delta\right\}
$$

for some sufficiently small $\delta$ and satisfying

$$
\int \delta_{b}=1
$$

The $b_{j}$ 's are chosen to be dependent on $\boldsymbol{a}$ and at the distance $\mathrm{O}(1)$ from but very close to the $a_{j}$ 's.

With the potential $\delta_{b}$ defined as above it is argued in [OSb] that $\mathcal{E}_{\lambda}(\psi)$ has a minimizer among functions with the given vortex configuration $(\boldsymbol{a}, \boldsymbol{n})$, provided

$$
\lambda_{j} \geqslant C\left|\nabla_{a_{j}} E(\boldsymbol{a})\right|
$$

for an appropriate constant $C=\mathrm{O}(1)$. Taking $\lambda=\mathrm{O}\left(a^{-1}\right)>$ constant $|\nabla E(c)|$, we obtain

$$
E(c) \geqslant \inf \left\{\mathcal{E}_{\lambda}(\psi) \mid \operatorname{conf} \psi=c\right\}-C \cdot a^{-1} .
$$

Now the minimizer, $\psi_{\lambda}$, of the variational problem on the r.h.s. satisfies the EulerLagrange equation

$$
-\Delta \psi+\left(|\psi|^{2}-1\right) \psi=-\Sigma \delta_{b_{j}} \psi .
$$

An elementary but somewhat lengthy analysis of this equation shows that its solutions satisfy estimates (2.11) and (2.12), i.e. are in the class considered before. Therefore

$$
\inf \left\{\mathcal{E}_{\lambda}(\psi) \mid \operatorname{conf} \psi=c\right\}=\text { r.h.s. (2.10). }
$$

This relation together with equation (2.19) implies the desired lower bound; the latter in turn together with the upper bound mentioned above yields (2.10).

One of the results described above states that if a field $\psi_{0}$ satisfies estimates (2.11) and (2.12), then

$$
\mathcal{E}_{\text {ren }}\left(\psi_{0}\right) \leqslant E(\boldsymbol{a})+\mathrm{O}\left(a^{-1}\right) .
$$

We conjecture that the opposite is also true: if $\psi_{0}$ satisfies inequality (2.21) (or a slightly stronger inequality) then $\psi_{0}$ satisfies estimates (2.11) and (2.12). 


\section{Nonlinear adiabatic theory}

Consider equations (1.1) and (1.2) with initial conditions $\left.\psi\right|_{t=0}=\psi_{0}$ having a given vortex configuration $\boldsymbol{c}_{0}=\left(\boldsymbol{a}_{0}, \boldsymbol{n}\right)$ with $a_{0} \equiv \min \left|a_{0 i}-a_{0 j}\right| \gg 1$ and of low energy:

$$
\mathcal{E}_{\text {ren }}\left(\psi_{0}\right) \leqslant E\left(a_{0}\right)+\mathrm{O}\left(a_{0}^{-1}\right) .
$$

We can think about the solutions $\psi$ as determined by two types of motion: a fast motion around vortex centres (with velocities $\sim\left|\nabla \psi^{\left(n_{i}\right)}\left(x_{0}\right)\right|,\left|x_{0}\right| \approx 1$ ) and a slow motion of the vortex centres themselves (with velocities $\left|\nabla_{a_{i}} E(\boldsymbol{a})\right|$ ). This suggests the use of an adiabatic approximation. In this approximation, at the first step the vortex centres are frozen and the lowest energy is found under this restriction. This energy is, of course, a function of the positions of vortex centres. On the second step, this energy is used as a Hamiltonian for the motion of the vortex centres.

Specifically, let $\psi_{a}$ be a differentiable family (depending on $\boldsymbol{a}$ ) of approximate minimizers of variational problem (2.9), s.t. $\psi_{a_{0}}=\psi_{0}$. Their energy is

$$
H_{1}(\boldsymbol{a})=\mathcal{E}_{\text {ren }}\left(\psi_{a}\right) .
$$

Use this energy as the Hamiltonian for the vortex centres

$$
\dot{\boldsymbol{a}}(t)=-J \nabla H_{1}(\boldsymbol{a}(t)),
$$

where $J$ is the same symplectic matrix as in (1.7), with the initial condition

$$
\boldsymbol{a}(0)=\boldsymbol{a}_{0},
$$

the vortex configuration of the initial condition, $\psi_{0}$, for (1.1) and (1.2). Solve equations (3.3) and (3.4) and form the adiabatic orbit

$$
\psi^{\text {adiab }}=\psi_{a(t)} .
$$

We will show below that $\psi^{\text {adiab }}$ solves equation (1.1) to the order $\mathrm{O}\left(a^{-2}\right)$. Moreover, we derive the estimate

$$
\sup _{x}\left|\psi(x, t)-\psi^{\text {adiab }}(x, t)\right| \leqslant C a^{-1},
$$

provided $t \leqslant a^{p}$ for some $p \geqslant 0$. We argue also that this estimate cannot be improved beyond $\mathrm{O}\left(a^{-2}\right)$. Indeed, the correction to $\psi^{\text {adiab }}$, which is of the order $\mathrm{O}\left(\frac{1}{a^{2}}\right)$ describes the process of radiation by the system of moving vortices and therefore cannot be incorporated into $\psi^{\text {adiab }}$ (see section 6).

Functions (3.5) are the main object of the adiabatic theory while the justification of inequality (3.6) or a variant of it is its main goal.

Initially there is unwelcome arbitrariness in the construction above: the choice of the family $\psi_{a}$ is not unique. However, as with the variational principle, this arbitrariness allows us a certain degree of flexibility. Moreover, due to (2.10), at least for the $\psi_{a}$ 's satisfying (2.11) and (2.12), different $\mathcal{E}\left(\psi_{a}\right)$ differ by $\mathrm{O}\left(a^{-1}\right)$ and these differences are absorbed by radiative excitations (see section 4$)$.

We point out that convenient choice of the family $\psi_{a}$ used in (3.2) is provided by the minimizer for the energy functional (2.14) for pinned vortices among functions with $\operatorname{conf} \psi=c$ (see $[\mathrm{OSb}]$ for more details).

The origin of the nonlinear adiabatic theory can be gleaned as follows. The action for equation (1.1) is

$$
S(\psi)=\int\left(-\frac{1}{2} \int \operatorname{Im} \psi \dot{\bar{\psi}}+\mathcal{E}_{\text {ren }}(\psi)\right) \mathrm{d} t .
$$


In order to find approximate stationary points of $S(\psi)$ we first find an approximate minimizer, $\psi_{\boldsymbol{a}}$, of the potential part $\mathcal{E}_{\text {ren }}(\psi)$ for the vortex centres frozen at $\boldsymbol{a}$ (so that $\partial_{\bar{\psi}} \mathcal{E}_{\text {ren }}\left(\psi_{a}\right)$ is very small, in fact $\mathrm{O}\left(a^{-2}\right)$ ). Then we allow $\boldsymbol{a}$ to be time dependent and plug $\psi_{a(t)}$ into the Lagrangian to obtain the effective action

$$
S_{\text {eff }}(\boldsymbol{a}):=S\left(\psi_{a}\right) .
$$

The theorem below shows that modulo $\int \mathrm{O}\left(\frac{\dot{a} \ln a}{a}\right) \mathrm{d} t$

$$
S_{\text {eff }}(\boldsymbol{a})=\int\left(-\frac{\pi}{2} \Sigma n_{j} \dot{a}_{j} \wedge a_{j}+H_{1}(\boldsymbol{a})\right) \mathrm{d} t .
$$

Equation (3.3) is an equation for stationary points of the action on the r.h.s..

Theorem 3.1. Let $\psi_{0}$ be a field satisfying estimates (2.11) and (2.12) (so $\operatorname{conf} \psi_{0}=\boldsymbol{c}$, $\boldsymbol{c}=(\boldsymbol{n}, \boldsymbol{a})$ ). Then $S\left(\psi_{0}\right)=$ r.h.s. (3.9) modulo $\int \mathrm{O}\left(\frac{\dot{a} \ln a}{a}\right) \mathrm{d}$. Moreover, the latter error can be presented as an explicit term of that order plus a term $\int \mathrm{O}\left(\frac{\dot{a}}{a}\right) \mathrm{d} t$.

A proof of this theorem is given in the appendix.

The nonlinear adiabatic theory is justified in sections 4-6.

\section{Effective action}

In order to justify the nonlinear adiabatic theory we present the desired solution, $\psi$, as $\psi=\psi_{a}+\alpha$, where $\alpha$ is a small fluctuation around the field $\psi_{a}$. Expanding the action $S(\psi)$ around $\psi_{a}$, we find modulo $\mathrm{O}\left(\alpha^{3}\right)$ that

$$
S(\psi)=S\left(\psi_{a}\right)+\operatorname{Re} \iint \bar{\alpha} \partial_{\bar{\psi}} S\left(\psi_{a}\right)+\frac{1}{2} \operatorname{Re} \iint \bar{\alpha} S^{\prime \prime}\left(\psi_{a}\right) \alpha .
$$

Here $S^{\prime \prime}\left(\psi_{a}\right)$ is the Hessian of $S$ or the linearized operator of (1.1) computed at $\psi_{a}$; it is defined by the relation

$$
\operatorname{Re} \iint \bar{\eta} S^{\prime \prime}(\psi) \xi=\left.\frac{\partial^{2}}{\partial \lambda \partial \mu} S\left(\psi_{\lambda \mu}\right)\right|_{\lambda=\mu=0},
$$

where $\psi_{\lambda \mu}=\psi+\lambda \xi+\mu \eta$.

The r.h.s. of (4.1) is a functional of $a(t)$ and $\alpha$. Critical points of this functional satisfy the equations (here we drop the term $\nabla_{a} \frac{1}{2} \operatorname{Re} \int \bar{\alpha} S^{\prime \prime}\left(\psi_{a}\right) \alpha$ )

$$
\begin{aligned}
& \partial_{\boldsymbol{a}} S\left(\psi_{\boldsymbol{a}}\right)=-\nabla_{\boldsymbol{a}} \operatorname{Re} \int \bar{\alpha} \partial_{\bar{\psi}} S\left(\psi_{\boldsymbol{a}}\right), \\
& S^{\prime \prime}\left(\psi_{a}\right) \alpha=-\partial_{\bar{\psi}} S\left(\psi_{a}\right) .
\end{aligned}
$$

The coupling between these two equations is weak. Indeed, we have the following.

Proposition 4.1. If $\psi_{\boldsymbol{a}}$ satisfies (2.11) and (2.12) and $\boldsymbol{a}(t)$ obeys equation (1.10), then

$$
\partial_{\bar{\psi}} S\left(\psi_{a}\right)=\mathrm{O}\left(a^{-2}\right) \text {. }
$$

Proof. First we remark that

$$
\partial_{\bar{\psi}} S(\psi)=-\Delta \psi+\left(|\psi|^{2}-1\right) \psi-\mathrm{i} \dot{\psi} .
$$

Applying to the r.h.s. equations (2.11) and (2.12), we obtain

$$
\partial_{\bar{\psi}} S\left(\psi_{a}\right)=\mathrm{ie}^{\mathrm{i} \varphi_{0}}\left(2 \nabla \psi_{j} \cdot \nabla \varphi_{(j)}+\dot{\varphi}_{j}\right)+\mathrm{O}\left(a^{-2}\right) .
$$


Since $\dot{\psi}_{j}=-\nabla \psi_{j} \cdot \dot{a}_{j}$ and, by equation (1.10), $\dot{a}_{j}=2 \nabla \varphi_{(j)}\left(a_{j}\right)$, and $\nabla \psi_{j}=\mathrm{O}\left(\left|x-a_{j}\right|^{-1}\right)$, we conclude that

$$
2 \nabla \psi_{j} \cdot \nabla \varphi_{(j)}+\dot{\psi}_{j}=2 \nabla \psi_{j}(x) \cdot\left(\nabla \varphi_{(j)}(x)-\nabla \varphi_{(j)}\left(a_{j}\right)\right)=\mathrm{O}\left(a^{-2}\right) .
$$

The proposition above suggests we set, in the leading order, the r.h.s. of (4.2) and (4.3) to zero. This results in equations (3.3) and $\alpha=0$. In the next approximation we plug the solution to equations (3.3) and (3.4) into the r.h.s. of (4.3), solve the resulting equation and plug its solution together with the mentioned solution to (3.3) and (3.4) into the r.h.s. of (4.2). The solution of the resulting equation together with the solution to (4.3) mentioned above yields the next approximation to $\boldsymbol{a}(t)$ and $\alpha$, and so on.

Equations (4.2) and (4.3) suggest that $\alpha=\mathrm{o}(1)$. This conclusion is confirmed in the next two sections by analysing more carefully equation (4.3).

The nature of the fluctuation field $\alpha$, in general, is rather complicated. For $\operatorname{dist}(x, \boldsymbol{a}) \ll$ $a^{2}, \alpha$ is essentially adiabatic, while for $\operatorname{dist}(x, \boldsymbol{a}) \geqslant \mathrm{O}\left(a^{2}\right), \alpha$ is a radiation field. Indeed, let $\Delta S$ be the sum of the last two terms on the r.h.s. of (4.1), the only terms containing $\alpha$. For $\operatorname{dist}(x, \boldsymbol{a}) \ll a^{2}$, the terms $\operatorname{Im}(\bar{\alpha} \cdot \dot{\alpha})$ and $\dot{\varphi}_{0}|\alpha|^{2}$ are of a higher order than, say, $2 \dot{\varphi}_{0} \operatorname{Re}\left(\bar{\psi}_{a} \alpha\right)$ and therefore can be dropped from $\Delta S$. Moreover we can drop the term $\operatorname{Im} \iint \frac{\mathrm{d}}{\mathrm{d} t}\left(\bar{\psi}_{a} \alpha\right)$, which is constant under the variations. The resulting action does not contain $\dot{\alpha}$. It leads to an elliptic equation for $\alpha$. This equation is studied in the next section where it is argued that it produces a unique localized solution of order $\mathrm{O}\left(a^{-1}\right)$.

Consider now the domain $D=\left\{x \in \mathbb{R}^{2} \mid \operatorname{dist}(x, \boldsymbol{a}) \gg a\right\}$. Since in $D$

$$
\left|\psi_{0}\right|=1+\mathrm{O}\left(d(x, \boldsymbol{a})^{-2}\right),
$$

it is convenient to incorporate $\left|\psi_{0}\right|-1$ into the fluctuation field and consider functions of the form

$$
\psi=\mathrm{e}^{\mathrm{i}\left(\varphi_{0}+\chi\right)}(1-\xi)
$$

with $0<\xi<1$. Now we plug this expression into $\Delta S(\psi)$ and omit the terms depending exclusively on $\varphi_{0}$ and its derivatives, the term $\int_{0}^{T} \int_{D_{0}} \dot{\chi}$ which is constant under variations and the term $\int_{0}^{T} \int_{D} \nabla \varphi_{0} \cdot \nabla \chi=\int_{0}^{T} \int_{\partial D} \frac{\partial \varphi_{0}}{\partial \nu} \chi$ determined by boundary conditions. As a result we arrive at

$$
\Delta S=\frac{1}{2} \iint\left(-2 \dot{\varphi}_{0} \xi-2 \dot{\chi} \xi+|\nabla \chi|^{2}+2 \xi^{2}\right),
$$

where we have also dropped the terms $\dot{\varphi}_{0} \xi^{2}$, and $|\nabla \xi|^{2}$ compared with the term $2 \xi^{2}$. The variation with respect to $\xi$ leads to the constraint

$$
\xi=\frac{1}{2}\left(\dot{\varphi}_{0}+\dot{\chi}\right),
$$

which we use in order to exclude $\xi$ from (4.9). As a result we arrive at the following correction to the action $S_{\text {eff }}(a) \equiv S\left(\psi_{a}\right)$

$$
\Delta S(\boldsymbol{a}, \chi)=\iint\left(-\frac{1}{2} \dot{\chi}^{2}+|\nabla \chi|^{2}+2 \ddot{\varphi}_{0} \chi-\dot{\varphi}_{0}^{2}\right) .
$$

The latter term is an action for a radiation field $\chi$ and its coupling to the vortex dynamics. Combining this term with the action $S_{\text {eff }}(\boldsymbol{a})$ and ignoring terms of the form $\Delta S(\boldsymbol{a})$ with higher order corrections to $S_{\text {vort }}(a) \equiv$ r.h.s. (3.10) which lead to standard corrections to the vortex law, we arrive at the following variational equations:

$$
\dot{\boldsymbol{a}}=J \nabla H_{1}(\boldsymbol{a})+2 \int \nabla_{\boldsymbol{a}} \varphi_{0} \ddot{\chi}
$$


and

$$
\left(\frac{1}{2} \partial_{t}^{2}-\Delta_{x}\right) \chi=-2 \ddot{\varphi}_{0} .
$$

These are coupled equations for motion of the vortex centres and radiation produced by this motion. They are idealizations of more precise equations (4.2) and (4.3).

\section{Method of geometric solvability}

In this section we analyse our main equation for the fluctuation field $\alpha$-equation (4.3). We present an argument that $\alpha=\mathrm{O}\left(a^{-1}\right)$. We solve this equation in the domains $\operatorname{dist}(x, \boldsymbol{a}) \leqslant r_{0}$, $r_{1} \leqslant \operatorname{dist}(x, \boldsymbol{a}) \leqslant R_{1}$ and $\operatorname{dist}(x, \boldsymbol{a}) \geqslant R_{2}$, where $r_{1}<r_{2} \ll a \ll R_{2}<R_{1}$, separately.

First we write out the operator $S^{\prime \prime}\left(\psi_{a}\right)$ entering the 1.h.s. of (4.3) explicitly

$$
S^{\prime \prime}\left(\psi_{a}\right) \alpha=-\mathrm{i} \dot{\alpha}-\Delta \alpha+\left(2\left|\psi_{a}\right|^{2}-1\right) \alpha+\psi_{a}^{2} \bar{\alpha} .
$$

For simplicity we take $\psi_{a}$ of the form $\psi_{a}=f_{0} \mathrm{e}^{\mathrm{i} \varphi_{0}}$, where $f_{0}=\left|\psi_{a}\right|$ and $\varphi_{0}$, recall, is defined in (2.13). Consequently, we look for $\alpha$ in the form

$$
\alpha=\mathrm{e}^{\mathrm{i} \varphi_{0}} \eta \text {. }
$$

Then equation (4.3) reduces to the following equation for $\eta$

$$
-\mathrm{i} \dot{\eta}+L(\eta)=F
$$

where $F=\mathrm{e}^{-\mathrm{i} \varphi_{0}} \partial_{\bar{\psi}} S\left(\psi_{a}\right)$ and $L$ is the operator given by

$$
L(\eta):=\left(-\Delta+\phi_{0}-2 \mathrm{i} \nabla \varphi_{0} \cdot \nabla\right) \eta+2 f_{0}^{2} \operatorname{Re} \eta
$$

with $\phi_{0}=\dot{\varphi}_{0}+\left|\nabla \varphi_{0}\right|^{2}+f_{0}^{2}-1$.

Our goal is to demonstrate that for $\boldsymbol{a}(t)$ satisfying equation (3.3), $\psi_{\boldsymbol{a}}$ can be adjusted by an $\mathrm{O}\left(a^{-2}\right)$ term in such a way that equation (5.1) has a unique, modulo zero modes of $L$, solution (for any appropriate initial condition) satisfying

$$
\eta=\mathrm{O}\left(a^{-1}\right)
$$

provided $t \leqslant a^{p}$ for some $p \geqslant 0$. In this section we consider the first two domains while the third one is treated in the next section.

(a) $D^{\prime} \equiv\left\{x \in \mathbb{R}^{2} \mid \operatorname{dist}(x, \boldsymbol{a}) \leqslant r_{0}\right\}, r_{0} \ll a$. The point here is that in this domain we can neglect the term $-\mathrm{i} \dot{\eta}$. Indeed, the time differentiation is of the order $\mathrm{O}\left(a^{-2}\right)$ so we throw $-\mathrm{i} \eta$ into the $\mathrm{O}\left(a^{-2}\right)$-error basket. Then the resulting equation is

$$
L(\eta)=F .
$$

First we note that the operator $L$, as well as the operators that follow, is self-adjoint in the inner-product

$$
\langle\eta, \xi\rangle=\operatorname{Re} \int \bar{\eta} \xi
$$

Next we find the (near) zero modes of $L$ and show that we can arrange by a small adjustment of the $\psi_{a}$ for the inhomogeneity, $F$, to be orthogonal to those modes. By the near zero modes we understand eigenfunctions with eigenvalues of o(1) on the scale of $a^{-1}$.

Note that $D^{\prime}$ is the union of the disks $D_{j}$ centred at $a_{j}$ and of the radius $r_{0}$. We show that in $D_{j}, L$ is unitary equivalent, modulo $\mathrm{O}\left(a^{-2}\right)$, to the linearization operator,

$$
L_{\psi_{j}}(\alpha)=\left(-\Delta+2\left|\psi_{j}\right|^{2}-1\right) \alpha+\psi_{j}^{2} \bar{\alpha}
$$

for the time-independent Ginzburg-Landau equation, equation (1.3), around $\psi_{j}$. Note that apart from the trivial shift $x \rightarrow x-a_{j}$, the operators $L_{\psi_{j}}$ are independent of $\boldsymbol{a}$. 
Lemma 5.1. If a(t) satisfies equation (3.3), then in $D_{j}$

$$
L=L_{j}+\mathrm{O}\left(a^{-2}\right),
$$

where the operator $L_{j}$ is defined by

$$
L_{j}(\eta):=\left(-\Delta+\left|\nabla \varphi_{j}\right|^{2}+f_{j}^{2}-1-2 i \nabla \varphi_{j} \cdot \nabla\right) \eta+2 f_{j}^{2} \operatorname{Re} \eta .
$$

(Note that apart from the trivial shift, $x \rightarrow x-a_{j}$, the operators $L_{j}$ are independent of $a$.)

Proof. Using that $\dot{a}_{j}=\mathrm{O}\left(a^{-1}\right)$ we compute in $D_{j}$

$$
\phi_{0}=\left|\nabla \varphi_{j}\right|^{2}+f_{j}^{2}-1+2 \nabla \varphi_{j} \cdot \nabla \varphi_{(j)}+\dot{\varphi}_{j}+\mathrm{O}\left(a^{-2}\right) .
$$

Due to the relation $\dot{\varphi}_{j}=-\nabla \varphi_{j} \cdot \dot{a}_{j}$, equation (3.3) and the estimate $\nabla \varphi_{j}=\mathrm{O}\left(\left|x-a_{j}\right|^{-1}\right.$ ), we have

$$
2 \nabla \varphi_{j} \cdot \nabla \varphi_{(j)}+\dot{\varphi}_{j}=\mathrm{O}\left(a^{-2}\right) .
$$

The last two estimates yield

$$
\phi_{0}=\left|\nabla \varphi_{j}\right|^{2}+f_{j}^{2}-1+\mathrm{O}\left(a^{-2}\right)
$$

in $D_{j}$, so (5.5) follows.

The last lemma states roughly that $L$ is an $\mathrm{O}\left(a^{-2}\right)$-perturbation of the direct sum $\bigoplus_{j=1}^{k} L_{j}$.

Now the operators $L_{j}$ are unitary equivalent to the operators $L_{\psi_{j}}$ :

$$
L_{j}(\eta)=\mathrm{e}^{-\mathrm{i} \varphi_{j}} L_{\psi_{j}}\left(\mathrm{e}^{\mathrm{i} \varphi_{j}} \eta\right) .
$$

It is shown in [OSa] that the only zero modes of the operator $L_{\psi_{j}}$ (this operator is independent of $a$ and therefore has no near zero modes) are those due to the breaking of the gauge symmetry, $\alpha_{0}^{(j)}=\mathrm{i} \psi_{j}$, and the translational symmetry $\alpha_{1}^{(j)}=\partial_{x_{1}} \psi_{j}$ and $\alpha_{2}^{(j)}=\partial_{x_{2}} \psi_{j}$. Due to (5.9), the only zero modes of $L_{j}$ are $\eta_{k}^{(j)}=\mathrm{e}^{-\mathrm{i} \varphi_{j}} \alpha_{k}^{(j)}, k=0,1,2$ (again $L_{j}$ has no near zero modes). Since $\eta_{0}^{(j)}$ does not decay at $\infty$ and $\eta_{1}^{(j)}$ and $\eta_{2}^{(j)}$ are 'essentially proportional' (see [OSa] for details) it suffices to consider only $\eta_{1}^{(j)}$.

Due to (5.5), the zero modes of $L_{j}, j=1, \ldots, k$, lead to near zero modes of $L$ with eigenvalues of order $\mathrm{O}\left(a^{-2}\right)$. These are the only (near) zero modes of $L$. Denote the (near) zero modes of $L$ corresponding to $\eta_{1}^{(j)}$ by $\eta^{(j)}$. They are localized essentially in $D_{j}$. Now the solvability conditions for equation (5.2) read (remember that $L$ is self-adjoint in the inner-product $\langle\xi, \eta\rangle=\operatorname{Re} \int \bar{\xi} \eta$ )

$$
\operatorname{Re} \int \bar{\eta}^{(j)} F=0
$$

$j=1, \ldots, K$. Since $F=\mathrm{O}\left(a^{-2}\right)$ and $\eta^{j}=\mathrm{O}(1)$, an $\mathrm{O}\left(a^{-2}\right)$ change in the original, input function $\psi_{a}$ suffices in order to satisfy this equation. (Here one can use an implicit function theorem in order to find such a correction.)

To sum up, we identified explicitly the near zero modes of the operator $L$ in equation (5.2) and argued that by an $\mathrm{O}\left(a^{-2}\right)$ perturbation of the input function $\psi_{a}$ the inhomogeneity $F$ can be brought to be orthogonal to those modes. Moreover, $L$ is an $\mathrm{O}\left(a^{-2}\right)$ perturbation of the direct sum, $\bigoplus_{j=1}^{K} L_{j}$, of $\boldsymbol{a}$-independent operators. Since $L_{j}$ are unitarily equivalent to $L_{\psi_{j}}$, their zero modes are explicitly known, they give rise to the near zero modes of $L$. Moreover, $L_{j} \geqslant 0$ if $n_{j}=1$ (see [OSa]). Since $L$ is defined on the union of disks, $D_{j}$, of radius $r_{0}$, generically there is a gap of order $\mathrm{O}\left(r_{0}^{-2}\right)$ between the near 
zero eigenvalues and the rest of the spectrum of $L$. As a result equation (5.2) has a unique solution, $\eta$, orthogonal to $\eta^{(1)}, \ldots, \eta^{(K)}$, and this solution is at least of the order

$$
\eta=\mathrm{O}\left(\frac{r_{0}^{2}}{a^{2}}\right)
$$

We believe that a more careful analysis based on considering, as in the next paragraph, the equations for the real and imaginary parts of $\eta$ separately yields the estimate

$$
\eta=\mathrm{O}\left(\frac{1}{a^{2}}\right)
$$

provided $r_{0} \leqslant a /(\ln a)^{2}$.

(b) $D^{\prime \prime}:=\left\{x \in \mathbb{R}^{2} \mid r_{1} \leqslant \operatorname{dist}(x, \boldsymbol{a}) \leqslant R_{1}\right\}, r_{1} \ll a \ll R_{1}$. This is a 'transitional' domain. Let $\eta=\eta_{1}+\mathrm{i} \eta_{2}$. In $D^{\prime \prime}$, we can solve equation (5.1) for $\eta_{1}$ approximately:

$$
\eta_{1} \approx \sum_{j=1}^{K} \nabla \varphi_{j}(x) \cdot\left(\nabla \varphi_{(j)}\left(a_{j}\right)-\nabla \varphi_{(j)}(x)\right)+\mathrm{O}\left(a^{-4}\right)+\mathrm{O}\left(a^{-2} \eta_{2}\right) .
$$

Here the leading term is of the order $\mathrm{O}\left(a^{-2}\right)$. Thus we have

$$
\eta_{1}=\mathrm{O}\left(a^{-2}\right)
$$

Now the equation for $\eta_{2}$ reads

$$
\left(-\Delta+\left|\nabla \varphi_{0}\right|^{2}+f_{0}^{2}-1+\dot{\varphi}_{0}\right) \eta_{2}=\dot{\eta}_{1}+2 \nabla \varphi_{0} \cdot \nabla \eta_{1}+\dot{f}_{0}+2 \nabla \varphi_{0} \cdot \nabla f_{0} .
$$

Since the $t$-derivative is of order $\mathrm{O}\left(a^{-2}\right)$ and the $x$-derivative in $D^{\prime \prime}$, of order $\mathrm{O}\left(r^{-1}\right)$, we conclude that the r.h.s. of (5.14) is of order $\mathrm{O}\left(r^{-2} a^{-2}\right)$. On the 1.h.s. we have as in the proof of lemma 5.1

$$
\begin{gathered}
\left|\nabla \varphi_{0}\right|^{2}+f_{0}^{2}-1+\dot{\varphi}_{0}=\sum_{j=1}^{K}\left(\left|\nabla \varphi_{j}\right|^{2}+f_{j}^{2}-1\right)+\sum_{j=1}^{K}\left(\dot{\varphi}_{j}+2 \nabla \varphi_{j} \cdot \nabla \varphi_{(j)}\right)+\mathrm{O}\left(a^{-4}\right) \\
=2 \sum_{j=1}^{K} \nabla \varphi_{j}(x) \cdot\left(\nabla \varphi_{(j)}\left(a_{j}\right)-\nabla \varphi_{(j)}(x)\right)+\mathrm{O}\left(r_{1}^{-4}\right)
\end{gathered}
$$

The leading term on the r.h.s. is of the order $\mathrm{O}\left(a^{-2}\right)$ and is essentially homogeneous in $a$ of degree -2 . If we rescale equation (5.14) as $x \rightarrow a^{2} x$, then we arrive at an equation of the form

$$
(-\Delta+V(x)) \eta_{2}^{\text {rescaled }}=\mathrm{O}\left(r^{-2} a^{-2}\right)
$$

on $a^{-1} D^{\prime \prime}$, with the potential term $V$ essentially independent of $a$. The operator $-\Delta+V(x)$ on $a^{-1} D^{\prime \prime}$ has a discrete spectrum with the gaps of order $\mathrm{O}\left(\frac{a^{2}}{R_{1}^{2}}\right)$. So for generic $V$ (which is fine with us since the input function $\psi_{a}$ can be adjusted) this equation has a unique solution of the order $\mathrm{O}\left(R_{1}^{2} a^{-2} r_{1}^{-2}\right)$. Thus we conclude that at least

$$
\eta=\mathrm{O}\left(R_{1}^{2} a^{-2} r_{1}^{-2}\right) \quad \text { in } D^{\prime \prime} .
$$




\section{Radiation}

In this section we consider equation (5.1) or the full equation, for the fluctuation field $\eta$ in the domain $D^{\prime \prime \prime}:=\left\{x \in \mathbb{R}^{2} \mid \operatorname{dist}(x, a) \geqslant R_{2}\right\}, R_{1}>R_{2} \gg a$, i.e. in the domain where we expect radiation to take place. This radiation is caused by moving vortices as was briefly pointed out in section 4.

We could start with equation (4.3) or (5.1) but instead we derive relevant equations from scratch, reproducing the conclusion arrived at the end of section 4 . The discussion in section 4 shows that the phase of $\psi$ dominates at large distances so we derive first an equation for it. Let $f=|\psi|$ and $\varphi=\arg \psi$ so that $\psi=f \mathrm{e}^{\mathrm{i} \varphi}$. Then equation (1.1) implies the following equations for $f$ and $\varphi$

$$
-\dot{\varphi}=-\frac{\Delta f}{f}+|\nabla \varphi|^{2}+f^{2}-1
$$

and

$$
\dot{f}=-\Delta \varphi f-2 \nabla \varphi \cdot \nabla f
$$

Solving the first of these equations for $f^{2}$ as

$$
f^{2}=1-|\nabla \varphi|^{2}-\dot{\varphi}+\frac{1}{f} \Delta f
$$

and substituting the result into the second equation, we find

$$
\ddot{\varphi}-2 \Delta \varphi=F,
$$

where

$F=-2 \nabla \varphi \cdot \nabla\left(|\nabla \varphi|^{2}+\frac{\partial \varphi}{\partial t}-\frac{1}{f} \Delta f\right)-2\left(1-f^{2}\right) \Delta \varphi-\frac{\partial}{\partial t}\left(|\nabla \varphi|^{2}-\frac{1}{f} \Delta f\right)$.

We solve equation (6.4) by perturbation theory. We look for $\varphi$ in the form

$$
\varphi=\varphi_{0}+\chi, \quad \text { where, recall, } \varphi_{0}=\sum_{j} n_{j} \theta\left(x-a_{j}(t)\right)
$$

with $\theta(x)$, the polar angle of $x \in \mathbb{R}^{2}$, and $\boldsymbol{a}(t)$, the solution to (1.10) with the initial condition $\boldsymbol{a}(0)$ given by the vortex configuration of the initial condition for the Schrödinger equation (1.1) (see equation (3.4)). Since $\Delta \varphi_{0}=0$, we have

$$
\ddot{\chi}-2 \Delta \chi=-\ddot{\varphi}_{0}+F \text {. }
$$

To estimate $F$ we plug in $\varphi_{0}$ for $\varphi$ and $f_{0}$ for $f$ into the r.h.s. of (6.5) to obtain

$$
F=\mathrm{O}\left(r^{-3} a^{-1}\right) \text {. }
$$

On the other hand, we compute

$\ddot{\varphi}_{0}=\sum_{j=1}^{K} n_{j} \frac{\left(x-a_{j}\right) \wedge \ddot{a}_{j}}{\left|x-a_{j}\right|^{2}}+\mathrm{O}\left(r^{-2} a^{-2}\right)=\sum_{j=1}^{K} n_{j} \frac{x \wedge \ddot{a}_{j}}{|x|^{2}}+\mathrm{O}\left(r^{-2} a^{-2}\right)$.

In passing to the last term on the r.h.s. we have used $|x| \gg\left|a_{j}\right| \forall j$. Since the centre of vorticity, $\Sigma n_{j} a_{j}$, is conserved in our approximation, we have $\Sigma n_{j} \ddot{a_{j}}=0$ and therefore

$$
\ddot{\varphi}_{0}=\mathrm{O}\left(r^{-2} a^{-2}\right) \text {. }
$$

Thus $F$ can be dropped from equation (6.7) and we arrive at

$$
\ddot{\chi}-2 \Delta \chi=-\ddot{\varphi}_{0}
$$


with zero initial conditions. Note that this equation also follows from varying action (4.11) with respect to the field $\chi$.

We ignore the boundary conditions since they contribute higher-order terms in $a^{-1}$.

We claim now that with $\tau=t / R_{1}^{2}$

$$
\chi=O\left(\frac{(\ln \tau)^{2}}{a^{2}}\right) .
$$

Indeed, rescaling equation (6.11) as $x \rightarrow y=\frac{x}{\sqrt{2} R_{1}}$ and $t \rightarrow \tau=\frac{t}{R_{1}^{2}}$ and applying Green's function method to the resulting equation, we find

$$
\chi^{\text {resc }}=-G * h,
$$

where $\chi^{\text {resc }}$ and $\ddot{\varphi}_{0}^{\text {resc }}$ denotes $\chi$ and $\ddot{\varphi}_{0}$ rescaled as indicated, $h=R_{1}^{2} \ddot{\varphi}_{0}^{\text {resc }}, *$ denotes the convolution in $y$ and $\tau$, and $G$ solves the equation

$$
\left(\frac{\partial^{2}}{\partial \tau^{2}}-\Delta\right) G=\delta(\tau) \delta(y)
$$

with the causality condition that $G=0$ for $\tau<0$. It can be found explicitly:

$$
G(\tau, y)=\frac{\chi_{\tau \geqslant \rho}}{2 \pi \sqrt{\tau^{2}-\rho^{2}}},
$$

where $\rho=|y|$ and $\chi_{\tau \geqslant 0}=1$ if $\tau \geqslant 0$ and $=0$ if $\tau<0$. Since $h=\mathrm{O}\left(a^{-2} \rho^{-2}\right)$, we estimate

$$
\left|\chi^{\mathrm{resc}}\right| \leqslant C a^{-2} \iiint_{\substack{|\Delta y| \leqslant s,\left|y^{\prime}\right| \geqslant 1 \\ 0 \leqslant s \leqslant \tau}} \frac{\mathrm{d}^{2} y^{\prime} \mathrm{d} s}{\left|y^{\prime}\right|^{2} \sqrt{s^{2}-|\Delta y|^{2}}},
$$

where $\Delta y:=y-y^{\prime}$.

First we let $\rho \geqslant 2 \tau$. In this case

$$
\left|y^{\prime}\right| \geqslant|y|-|\Delta y| \geqslant \frac{1}{2} \rho .
$$

So the triple integral on the r.h.s., which we denote by $I$, is estimated as

$$
I \leqslant \frac{4}{\rho^{2}} \iint_{|z| \leqslant \tau} \mathrm{d}^{2} z \int_{|z|}^{\tau} \frac{\mathrm{d} s}{\sqrt{s^{2}-|z|^{2}}} \leqslant \frac{8 \sqrt{\tau}}{\rho^{2}} \iint_{|z| \leqslant \tau} \frac{\mathrm{d}^{2} z}{\sqrt{|z|}} \leqslant \text { constant } .
$$

Here we made the change of the variable of integration as $y^{\prime} \rightarrow z=y-y^{\prime}=\Delta y$.

Now we let $\rho \leqslant 2 \tau$. We decompose the triple integral $I$ into the sum of the three integrals, $I_{1}, I_{2}$ and $I_{3}$, defined by the following additional restrictions on the domain of integration:

$$
s \leqslant 1 ; s \geqslant 1,|\Delta y| \leqslant \frac{1}{2} s \quad \text { and } \quad|\Delta y| \geqslant \frac{1}{2} s \geqslant \frac{1}{2}
$$

respectively. Since $\left|y^{\prime}\right| \leqslant|y|+|\Delta y| \leqslant 3 \tau$, the first integral is estimated as

$$
I_{1} \leqslant \iint_{1 \leqslant\left|y^{\prime}\right| \leqslant 3 \tau} \frac{\mathrm{d}^{2} y^{\prime}}{\left|y^{\prime}\right|^{2}} \int_{|\Delta y|}^{1} \frac{\mathrm{d} s}{\sqrt{s^{2}-|\Delta y|^{2}}} \leqslant \text { constant. }
$$

For the second integral we obtain inequality

$$
I_{2} \leqslant 2 \int_{1}^{\tau} \frac{\mathrm{d} s}{s} \int_{1 \leqslant\left|y^{\prime}\right| \leqslant 3 \tau} \frac{\mathrm{d}^{2} y^{\prime}}{\left|y^{\prime}\right|^{2}} \leqslant \text { constant } \cdot(\ln \tau)^{2} .
$$


Finally, the third integral is estimated as follows

$$
I_{3} \leqslant \int_{1 \leqslant\left|y^{\prime}\right| \leqslant 3 \tau} \frac{\mathrm{d}^{2} y^{\prime}}{\left|y^{\prime}\right|^{2}} \int_{|\Delta y|}^{2|\Delta y|} \frac{\mathrm{d} s}{\sqrt{s^{2}-|\Delta y|^{2}}} \leqslant \text { constant } \ln \tau .
$$

Collecting estimates (6.17)-(6.19) we arrive at the inequality

$$
I \leqslant \text { constant } \cdot(\ln \tau)^{2} .
$$

This together with (6.16) shows that estimate (6.20) always holds, which, in turn, together with (6.15) implies that

$$
\left|\chi^{\text {resc }}\right| \leqslant \text { constant } \cdot a^{-2} \cdot(\ln \tau)^{2} .
$$

Thus (6.12) follows.

On the other hand, equations (6.3) and (6.6) imply that

$$
1-f^{2}=\mathrm{O}\left(R_{2}^{-2}\right) \ll|\chi|,
$$

which justifies our idea that the phase plays a dominant role in the radiation process.

By the definition of $\eta$ (see the beginning of section 5), $\eta=f \mathrm{e}^{-\mathrm{i} \chi}-f_{0}$. Hence estimates (6.12) and (6.21) imply that for $t \leqslant a^{p}$ for some $p \geqslant 0$

$$
\eta=\mathrm{O}\left(a^{-2}(\ln a)^{2}\right) \quad \text { in } D^{\prime \prime \prime} .
$$

Combining estimates (5.12), (5.18) and (6.22) and setting $r_{0}, r_{1} \asymp \sqrt{R_{1}}$ and $R_{1}, R_{2} \asymp a$, we arrive at the estimate

$$
\eta=\mathrm{O}\left(a^{-1}\right)
$$

which, due to the relation $\psi-\psi^{\text {adiab }}=\mathrm{e}^{\mathrm{i} \varphi_{0}} \eta$, implies (3.6), provided $t \leqslant a^{p}$ for some $p \geqslant 0$.

\section{Solitary wave}

Equation (6.4) (or equations (6.1) and (6.2)) has, in a leading order in $\frac{1}{a}$, also a solitary wave solution. Indeed, retaining in (6.5) only leading terms, we obtain

$$
\left(\frac{\partial^{2}}{\partial t^{2}}-2 \Delta\right) \varphi=-2 \frac{\partial}{\partial t}|\nabla \varphi|^{2}-2 \frac{\partial \varphi}{\partial t} \Delta \varphi-\frac{1}{2} \frac{\partial^{2}}{\partial t^{2}} \Delta \varphi .
$$

This equation has a solution of the form $\varphi\left(x_{1}-v t\right)$, where $\alpha(z)=\frac{\partial \varphi}{\partial z}$ satisfies the equation

$$
\left(v^{2}-2\right) \alpha=3 v \alpha^{2}-\frac{v^{2}}{2} \alpha^{\prime \prime} \text {. }
$$

This equation with the boundary conditions $\alpha \rightarrow 0$ as $z \rightarrow \pm \infty$ is equivalent to the equation

$$
\left(v^{2}-2\right) \alpha^{2}-2 v \alpha^{3}+\frac{v^{2}}{2}\left(\alpha^{\prime}\right)^{2}=0,
$$

whose solution is

$$
\alpha=-\frac{A}{\cosh ^{2} \beta z},
$$

where

$$
\beta^{2}=\frac{2-v^{2}}{2 v^{2}} \quad \text { and } \quad A=\frac{2-v^{2}}{2 v} .
$$

Equations (7.1) and (7.3) are valid only for

$$
0<\sqrt{2}-v \ll 1 \text {. }
$$




\section{Radiation by two 1-vortices}

The purpose of this section is twofold. On one hand we describe the radiation and its effect on the vortex motion explicitly in the simplest case of two 1-vortices. On the other hand, it is the first step in understanding the large time vortex dynamics in $\mathbb{R}^{2}$. Indeed, as we argued in the previous section moving vortices, in general, radiate. As a result they lose their energy so that vortices of same sign move apart from each other, while those of opposite signs move closer (and eventually collapse onto each other). So the question is how the dynamics looks asymptotically and the natural guess is that it is a superposition of simple motions of well-separated vortices, the simplest of which is considered in this section.

In this section we describe the results and some of the ideas of their derivation. The details as well as a treatment of the dynamics of the vortex-antivortex system are given in the companion paper [OSe].

We consider equation (1.1) with an initial condition $\psi_{0}$ describing two 1-vortices at the distance $2 a \gg 1$ from one another. (Note that we changed here the definition of $a$ compared with the previous sections: $a \rightarrow \frac{a}{2}$.) Then in the adiabatic approximation, equation (1.10), the vortices rotate around their 'centre of vorticity', $\frac{1}{2}\left(1 \cdot a_{1}+1 \cdot a_{2}\right)=\frac{1}{2}\left(a_{1}+a_{2}\right)$, with the frequency

$$
\omega=\frac{1}{a^{2}} .
$$

Since the centre of vorticity is conserved under the evolution (1.7),

$$
\sum n_{j} a_{j}(t)=\text { constant }
$$

we can place it into the origin, so that $a_{1}= \pm a_{2}$ and can be written, modulo a constant phase shift due to the initial conditions, as

$$
a_{1,2}= \pm a(\cos \omega t, \sin \omega t) .
$$

Next we consider the equation for the radiation, equation (6.11). Using (8.3) and definition (6.8) of $\varphi_{0}$, we obtain

$$
\dot{\varphi}_{0}=2 a^{2} \omega \frac{a^{2}-r^{2} \cos 2(\theta-\omega t)}{a^{4}+r^{4}-2 r^{2} a^{2} \cos 2(\theta-\omega t)} .
$$

Thus the phase $\chi$ satisfies wave equation (6.11) with a source in the r.h.s. which depends on $r, a, \theta$ and $t$, only in the combinations $\frac{a^{2}}{r^{2}}$ and $\cos 2(\theta-\omega t)$ and is a periodic and even function of the second variable. Such a source generates a wave which for $r \gg 1$ has the form (see $[\mathrm{OSe}]$ )

$$
\chi=-\frac{(\pi \omega)^{1 / 2} \omega a^{2}}{2^{3 / 4} r^{1 / 2}} \cos \left[\sqrt{2} \omega(r-\sqrt{2} t)+2 \theta-\frac{\pi}{4}\right]
$$

plus higher harmonics, with the $n$th harmonic being of the order $\mathrm{O}\left(\sqrt{\frac{\omega}{2}}(\omega a)^{2 n}\right)$. Then the energy-conservation law implies that the distance, $2 a$, between vortices must increase in time.

In order to find the dependence of $a=a(t)$ on $t$ we compute the average $\left\langle S_{\psi}\right\rangle$ of the energy flux density,

$$
S_{\psi}=-\operatorname{Re}(\dot{\psi} \nabla \bar{\psi})=-\dot{\chi} \nabla \chi
$$

over the period, $\frac{\pi}{\omega}$, of the adiabatic motion of the vortices. Using (8.5), we find

$$
\left\langle S_{\psi}\right\rangle=\frac{\pi}{2 a^{6}} \frac{x}{|x|^{2}} .
$$


Then the energy conservation law

$$
\frac{\partial}{\partial t} \int_{\Omega} e(\psi)=\int_{\partial \Omega} S_{\psi}
$$

where $e(\psi)=\frac{1}{2}|\nabla \psi|^{2}+\frac{1}{4}\left(|\psi|^{2}-1\right)^{2}$, the energy density, implies that

$$
\frac{\mathrm{d} a}{\mathrm{~d} t}=\frac{\pi}{2 a^{5}} \text {. }
$$

Integrating this equation we find

$$
a(t)^{6}=a(0)^{6}+3 \pi\left(t-t_{0}\right),
$$

i.e. $a(t)$ grows with time as $(3 \pi t)^{\frac{1}{6}}$. See [OSe] for more details.

\section{Acknowledgments}

The authors are grateful to Weinan E and Stephen Gustafson for useful discussions and to the referee for the very prescient remarks which led to an improvement of this paper. Research on this paper was supported by NSERC under grant NA7901.

\section{Appendix. Proof of theorem 3.1}

Although one can analyse $\int \operatorname{Im}\left(\psi_{0} \dot{\bar{\psi}}_{0}\right)$ using estimates (2.11) and (2.12) directly it is simpler to use the polar representation for $\psi_{0}$. Let $f=\left|\psi_{0}\right|$ and $\varphi=\arg \psi_{0}$ so that $\psi=f \mathrm{e}^{\mathrm{i} \varphi}$. Then

$$
-\int \operatorname{Im}\left(\psi_{0} \dot{\bar{\psi}}_{0}\right)=\frac{1}{2} \int f^{2} \dot{\varphi}=\frac{1}{2} \int \dot{\varphi}-\frac{1}{2} \int\left(1-f^{2}\right) \dot{\varphi} .
$$

Here $\int \dot{\varphi}$ is understood as $\lim _{R \rightarrow \infty} \int_{|x| \leqslant R} \dot{\varphi}$. Proceeding as with the estimation of $E(\boldsymbol{a})$ in [OSb] (by decomposing the integral over disks, $D_{j}$, of the radii $r_{0}$ around the $a_{j}$ 's and over the rest and using (2.11) in $D_{j}$ and (2.12), in $\mathbb{R}^{2} \backslash \bigcup_{j} D_{j}$ ), we obtain

$\frac{1}{2} \int\left(1-f^{2}\right) \dot{\varphi}=-\pi \sum_{j \neq k} n_{k} n_{j}^{2} \dot{a}_{j k} \wedge \frac{a_{j k}}{\left|a_{j k}\right|^{2}} \ln \left|a_{j k}\right|+\sum \mathrm{O}\left(\frac{\dot{a}_{j}}{a}\right)$,

where $a_{j k}=a_{j}-a_{k}$ and $a \wedge b=a_{1} b_{2}-a_{2} b_{1}$.

In the first term on the r.h.s. of (A.1), we replace $\dot{\varphi}$ by $\dot{\varphi}_{0}$ since $\int \frac{\mathrm{d}}{\mathrm{d} t}\left(\varphi-\varphi_{0}\right)$ does not change under variations. Then writing $\dot{\varphi}_{0}=-\Sigma \nabla \varphi_{j} \cdot \dot{a}_{j}$ and choosing in the integral $\int \nabla \varphi_{j} \cdot \dot{a}_{j} \cdot d^{2} x$ the $x_{1}$-axis along $a_{j}$, we compute

$$
\frac{1}{2} \int_{|x| \leqslant R} \dot{\varphi}_{0}=\sum_{j} \frac{n_{j}}{2\left|a_{j}\right|} \dot{a}_{j} \wedge a_{j} \int_{0}^{R} I(r) r \mathrm{~d} r
$$

where $I(r)=\int_{0}^{2 \pi} \frac{r \cos \theta-\left|a_{j}\right|}{r^{2}+a_{j}^{2}-2 r\left|a_{j}\right| \cos \theta} \mathrm{d} \theta$. Next, we find

$$
\begin{gathered}
I(r)=-\frac{1}{2 i\left|a_{j}\right|}\left\{2 \pi i+\frac{r^{2}-a_{j}^{2}}{\left|a_{j}\right| r} \oint \frac{\mathrm{d} z}{\left(z-\frac{r}{\left|a_{j}\right|}\right)\left(z-\frac{\left|a_{j}\right|}{r}\right)}\right\} \\
= \begin{cases}0 & \text { if } r>\left|a_{j}\right| \\
-\frac{2 \pi}{\left|a_{j}\right|} & \text { if } r<\left|a_{j}\right| .\end{cases}
\end{gathered}
$$


The last two relations yield

$$
\frac{1}{2} \int \dot{\varphi}_{0}=-\frac{\pi}{2} \sum_{j} n_{j} \dot{a}_{j} \wedge a_{j} .
$$

Combining this with equations (A.1) and (A.2) and taking the $R \rightarrow \infty$ limit, we arrive at

$$
-\int \operatorname{Im}\left(\psi_{0} \dot{\bar{\psi}}_{0}\right)=-\frac{\pi}{2} \sum_{j} n_{j} \dot{a}_{j} \wedge a_{j},
$$

modulo $\sum_{j \neq k} \mathrm{O}\left(\frac{\ln \left|a_{j k}\right|}{\left|a_{j k}\right|} \dot{a}_{j k}\right)$. This together with the relation $\mathcal{E}_{\text {ren }}\left(\psi_{0}\right)=H_{1}(\boldsymbol{a})$ yields

$$
\mathcal{S}\left(\psi_{0}\right)=\int\left(-\frac{\pi}{2} \Sigma n_{j} \dot{a}_{j} \wedge a_{j}+H_{1}(\boldsymbol{a})\right) \mathrm{d} t .
$$

\section{References}

[AH] Atiyah M and Hitchin N 1988 The Geometry and Dynamics of Magnetic Monopoles (Princeton, NJ: Princeton University Press)

[BBH] Bethuel F, Brezis H and Hélein F 1994 Ginzburg-Landau Vortices (Basel: Birkhäuser)

[CK] Chanillo S and Kiesling M 1995 Symmetry of solutions of Ginzburg-Landau equations C. R. Acad. Sci. Paris 327 1023-6

[CM] Creswick J and Morrison N 1980 On the dynamics of quantum vortices Phys. Lett. 76A 267

[FS] Fröhlich J and Struwe M 1990 Variational problems on vector bundles Commun. Math. Phys. 131 431-64

[G] Gross E 1966 Dynamics of interacting bosons Physics of Many Particle Systems ed E Meeron (New York: Gordon and Breach) p 268

[H] Hagan P 1982 Spiral waves in reaction diffusion equations SIAM J. Appl. Math. 42 762-86

[JT] Jaffe A and Taubes C 1980 Vortices and Monopoles (Basel: Birkhäuser)

[Ma] Manton N S 1981 A remark on scattering of BPS monopoles Phys. Lett. 110B 54-6

[LL] Lieb E M and Loss M Symmetry of the Ginzburg-Landau minimizers in a disc Math. Res. Lett. 1 701-15

[Lin] Lin F H 1996 Some dynamical properties of Ginzburg-Landau vortices Commun. Pure Appl. Math. 49 323-59

[M1] Mironescu P 1995 On the stability of radial solutions of the Ginzburg-Landau equation J. Funct. Anal. $130334-44$

[M2] Mironescu P 1996 Les minimiseurs locaux pour l'équation de Ginzburg-Landau sont à symmétrie radiale C. R. Acad. Sci., Paris 323 593-8

[N] Neu J 1990 Vortices in complex scalar fields 1990 Physica D 43 385-406

[O] Onsager L 1949 Statistical hydrodynamics Nuovo Cimento V-VI suppl 2279

[OSa] Ovchinnikov Yu N and Sigal I M 1997 Ginzburg-Landau equation I. General discussion (P.D.E.s and their applications) CRM Proceedings and Lecture Notes vol 12 ed P Greiner et al (Providence, RI: American Mathematical Society)

[OSb] Ovchinnikov Yu N and Sigal I M 1998 The Ginzburg-Landau equation II. The energy of vortex configurations Physica D to appear

[OSc] Ovchinnikov Yu N and Sigal I M 1996 Symmetry breaking in the Ginzburg-Landau equation Preprint

[OSe] Ovchinnikov Yu N and Sigal I M 1998 Long-time behaviour of Ginzburg-Landau vortices Nonlinearity 11 1277-91

[S] Shafrir I 1994 Remarks on solutions of $-\Delta u=\left(1-|u|^{2}\right) u$ in $\mathbb{R}^{2}$ C. R. Acad. Sci. Paris 318 327-31

[Sh] Shah P A 1994 Vortex scattering at near critical coupling Nucl. Phys. B 429 259-76

[Stu] Stuart D 1994 Commun. Math. Phys. 15951 